\title{
Reacciones adversas a la transfusión de componentes sanguíneos en el sureste de México
}

\author{
Roberto Alejandro Sánchez-González ${ }^{*}$, Minerva Daniela Rodríguez-Parra², María Guadalupe \\ Trujillo-Vizuet ${ }^{1}$, Gisel Aracely Magaña-Pinto ${ }^{1}$ y Fabiola Yeseline Zamudio-Castellanos ${ }^{1}$ \\ ${ }^{1}$ Laboratorio de Investigación; ${ }^{2}$ Servicio Transfusional. Hospital Regional de Alta Especialidad Ciudad Salud, Tapachula, Chp., México
}

\section{RESUMEN}

Introducción: Las reacciones adversas a la transfusión (RAT) se definen como la aparición de cualquier evento adverso durante o después de la transfusión de sangre o de cualquiera de sus componentes. Objetivo: Determinar la prevalencia de reacciones adversas postransfusionales a los componentes sanguíneos administrados en el Hospital Regional de Alta Especialidad Ciudad Salud, en el periodo comprendido entre 2013 y 2017. Métodos: Se realizó un estudio descriptivo y retrospectivo. Se revisaron los reportes de reacciones transfusionales del periodo de estudio, y las reacciones se clasificaron de acuerdo con los estándares y definiciones reconocidas por la Asociación Americana de Bancos de Sangre (AABB). Resultados: Se reportaron un total de 38 RAT, con una prevalencia del $0.38 \%$. El concentrado eritrocitario fue el componente sanguíneo que presentó más RAT, con un 55.3\%. La reacción alérgica fue el tipo de reacción que se presentó con mayor frecuencia, con un 60.5\% $(n=23)$.

Palabras clave: Reacciones adversas. Componente sanguíneo. Hemovigilancia.

\begin{abstract}
Background. Adverse reactions to transfusion are defined as the occurrence of any adverse event during or after the transfusion of blood or any of its components. Objective: To determine the prevalence of post-transfusional adverse reactions to the blood components administered at the High-Specialty Regional Hospital of "Ciudad Salud", in Chiapas, Mexico, in the period 2013 to 2017. Methods: A descriptive and retrospective study was conducted. The reports of the transfusion reactions occurred during the study period were reviewed. The reactions were classified according to the standards and recommendations recognized by the American Association of Blood Banks. Results: A total of 38 adverse transfusion reactions were reported, with a prevalence of $0.38 \%$; the erythrocyte concentrate was the blood component that elicited the most adverse reactions to transfusion, in 55.3\%. The allergic reaction was the most frequent type of reaction, in 60.5\% $(n=23)$. (Hosp Med Clin Manag. 2018;11:126-9)

Corresponding author: Roberto Alejandro Sánchez-González, roasago@gmail.com
\end{abstract}

Key words: Adverse reactions. Blood component. Hemovigilance.

\section{Correspondencia:}

*Roberto Alejandro Sánchez-González

Hospital Regional de Alta Especialidad Ciudad Salud

Carretera Puerto Madero, s/n, km $15+200$

Col. Los Toros, Puerto Madero

Date of reception: 05-07-2018

C.P. 30830, Tapachula, Chp., México

Date of acceptance: 31-08-2018

E-mail: roasago@gmail.com

Doi: 10.24875/HMCM.18000140 


\section{INTRODUCCIÓN}

La transfusión sanguínea es la infusión de sangre entera o de componentes sanguíneos (como el plasma, el concentrado de plaquetas o eritrocitos) en la corriente sanguínea para reemplazar la pérdida sanguínea producida por un traumatismo, intervención quirúrgica o enfermedad ${ }^{1}$. La administración de hemocomponentes se puede infundir en el receptor a partir de un donante con el grupo sanguíneo y subgrupos antigénicos $A B O$ compatibles y concentraciones adecuadas de hemoglobina ${ }^{2}$

La transfusión de productos sanguíneos es una herramienta valiosa pero que debe usarse con prudencia, ya que se ha reportado que ocasiona ciertas reacciones adversas que llegan a ser fatales ${ }^{1,2}$.

El uso apropiado de la sangre y componentes sanguíneos se define como la transfusión de productos de la sangre seguros, y se emplea para tratar aquellas condiciones que pueden llevar a morbilidad significativa y/o mortalidad y que no pueden ser prevenidas o manejadas efectivamente por ningún otro medio ${ }^{3}$.

Los efectos indeseables o reacciones adversas con la administración de diferentes componentes sanguíneos se conocen como reacciones adversas asociadas a la transfusión (RAT) ${ }^{4}$. Las RAT se definen como la aparición de cualquier evento adverso durante o después de la transfusión de sangre o de cualquiera de sus componentes. Según el intervalo de tiempo entre la transfusión y la presentación de reacciones adversas se clasifican como: a) agudas, aquéllas que se presentan durante o antes de $24 \mathrm{~h}$, y b) como diferidas, aquéllas que suceden después de $24 \mathrm{~h}$. De acuerdo a los mecanismos fisiopatogénicos que intervienen en su producción, las RAT se clasifican en: inmunes y no inmunes o también como infecciosas y no infecciosas. En la tabla 1 se describe la clasificación de las RAT ${ }^{5-7}$.

Existen pocos datos en México sobre las RAT. El Hospital General de México reportó una tasa de 1.7 por cada 1,000 transfusiones, y Linares, en 2014, reportó un $0.3 \%$ de RAT ${ }^{7}$. Debido a ello, es de suma importancia conocer la prevalencia de las reacciones adversas, pues permitiría mejorar el uso de los hemocomponentes y controlar las reacciones adversas que se puedan presentar ${ }^{7,8}$.
Tabla 1. Clasificación de las RAT $^{7}$

\begin{tabular}{l}
\hline Reacciones \\
\hline Inmunológicas \\
\hline Inmediatas \\
- Hemolítica \\
- Febril no hemolítica \\
- Alérgicas: \\
- Urticaria \\
- Anafilaxia \\
- Daño pulmonar agudo asociado a la transfusión \\
Tardías \\
- Aloinmunización contra antígenos eritrocitarios, leucocitarios, \\
plaquetarios o proteínas plasmáticas \\
- Hemolítica \\
- Enfermedad injerto contra hospedero \\
- Púrpura postransfusión \\
- Inmunomodulación por transfusión \\
\hline No inmunológicas \\
\hline Inmediatas \\
- Contaminación bacteriana \\
- Sobrecarga circulatoria \\
- Hemólisis no inmune \\
- Embolia \\
- Hipotermia \\
- Desequilibrio hidroelectrolítico (hipocalcemia, hiperpotasemia, \\
hipomagnesemia) \\
- Coagulopatía hemodilucional \\
Tardías \\
- Hemosiderosis \\
- Transmisión de infecciones virales, bacterianas y parasitarias \\
\hline
\end{tabular}

El objetivo de este estudio fue determinar la prevalencia de reacciones adversas postransfusionales a los componentes sanguíneos administrados en el Hospital Regional de Alta Especialidad Ciudad Salud, de acuerdo a los reportes comprendidos entre los años 2013 y 2017.

\section{MÉTODOS}

Se realizó un estudio descriptivo y retrospectivo. Se revisaron 9,814 reportes de reacciones transfusionales que fueron llevadas en el Servicio Transfusional del hospital de tercer nivel Hospital Regional de Alta Especialidad Ciudad Salud en el periodo comprendido entre enero de 2013 y diciembre de 2017. Fueron incluidos todos los reportes para poder realizar el análisis de manera más completa. Las reacciones se clasificaron de acuerdo con los estándares y definiciones reconocidas por la AABB. Se analizaron las siguientes variables: hemocomponentes transfundidos, sexo, tipos de reacción y grupo sanguíneo de los receptores. El 
análisis de datos se realizó en el software de IBM SPSS, versión $23^{9}$.

Este estudio está registrado ante los comités de investigación y ética en investigación del hospital con el número de registro 04/2018.

\section{RESULTADOS}

Se administraron 9,814 componentes sanguíneos, y la prevalencia total de RAT fue del $0.38 \%$. El componente más transfundido fue el concentrado eritrocitario, con un $68.46 \%$ ( $n=6,719)$, seguido del plasma fresco congelado, con un $19.21 \%(n=1,886)$. La distribución por sexo de los pacientes que presentaron RAT fue: $55.3 \%$ $(n=21)$ mujeres y $44.7 \%(n=17)$ varones. El componente sanguíneo con más RAT fue el concentrado eritrocitario, con un $55.3 \%(n=21)$, seguido por plasma fresco congelado, con un $39.5 \%(n=15)$, y el concentrado plaquetario, con un $5.3 \%(n=2)$.

El tipo de reacción con mayor frecuencia fueron las alérgicas, con un $60.5 \%(n=23)$, seguidas por las reacciones febriles no hemolíticas, con un 26.3\% ( $n=10)$, las reacciones hemolíticas, con un $10.5 \%(n=4)$, y las bacterianas, con un 2.6\% $(n=1)$ (Fig. 1).

Con respecto a los tipos sanguíneos de los receptores que presentaron RAT, se encontró con mayor porcentaje en los pacientes con grupo O, con un $84.2 \%$, ( $n=$ 32 ), y en menor porcentaje en los de los grupos A1, A2 y B (Tabla 2).

\section{DISCUSIÓN}

A pesar de los estrictos controles que se llevan a cabo para realizar una transfusión sanguínea, los receptores pueden presentar efectos no deseados. Se transfundieron un total de 9,814 unidades de componentes sanguíneos durante el periodo de estudio, en las cuales se presentaron un $0.38 \%(n=38)$ de reacciones transfusionales, en comparación con el estudio realizado por Linares, donde se encontró un $0.3 \%$ de reacciones transfusionales ${ }^{7}$. A pesar de ello, los datos son más bajos a los reportados en 2007, con una tasa anual del $1.7 \% 8$. Estudios realizados en otros países reportan prevalencias diferentes, con un $0.16^{10}, 0.20^{11}$ y $1.2 \%{ }^{12}$

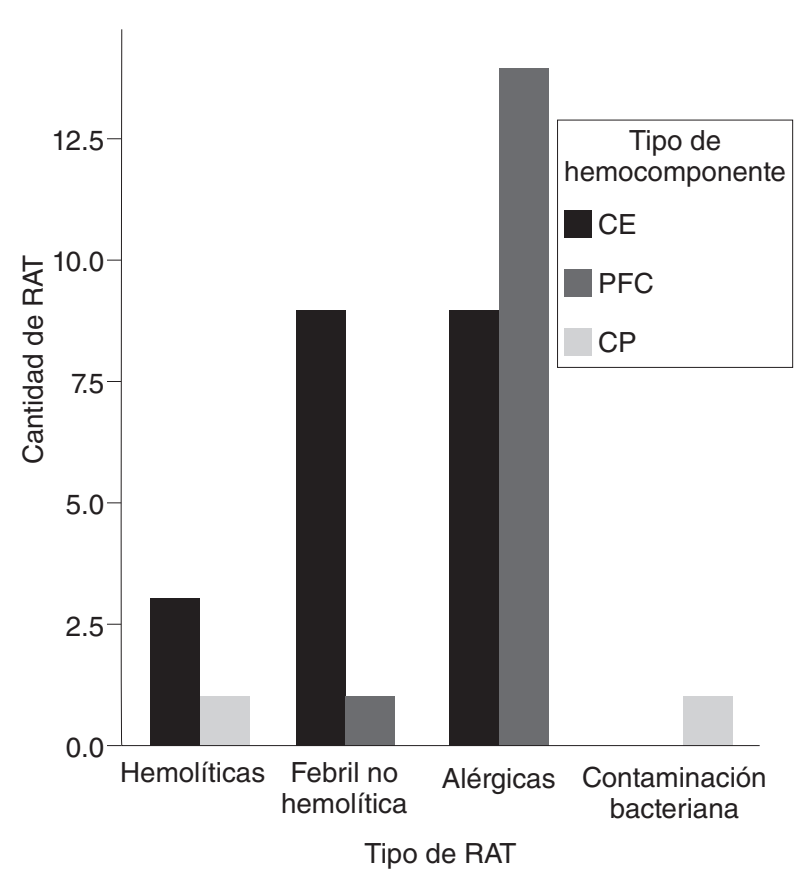

Figura 1. Tipo de reacción adversa y tipo de hemocomponente. CE: concentrado eritrocitario; PFC: plasma fresco congelado; $\mathrm{CP}$ : concentrado plaquetario.

Tabla 2. Frecuencia de grupo sanguíneo de los receptores

\begin{tabular}{lcc}
\hline Grupo sanguíneo & Frecuencia & Porcentaje \\
\hline 0 & 32 & 84.2 \\
A1 & 3 & 7.9 \\
A2 & 1 & 2.6 \\
B & 2 & 5.3 \\
Total & 38 & 100 \\
\hline
\end{tabular}

El tipo de reacción que con mayor frecuencia encontramos fueron las alérgicas, con un $60.5 \%(n=23)$, seguidas por las febriles no hemolíticas, con un $26.3 \%$ $(n=10)$. Estos porcentajes difieren de los reportados por Philip y Dimri ${ }^{13}$, que encontraron un $51.4 \%$ de reacciones febriles no hemolíticas y un $40.14 \%$ de reacciones alérgicas.

Las personas del sexo femenino fueron las que más RAT presentaron, al igual que en el estudio de Gutiérrez $^{8}$, que reportó al sexo femenino con más probabilidades de padecer RAT.

También se encontró que los concentrados eritrocitarios son los componentes sanguíneos más administrados, seguidos del plasma fresco congelado $y$, en menor frecuencia, el concentrado plaquetario, al igual que los 
componentes administrados en el estudio realizado por Philip y Dimri ${ }^{13}$, y a diferencia del de Linares, que encontró en segundo lugar al concentrado plaquetario?.

Se encontró una RAT por contaminación bacteriana. Esto es poco común y se atribuye generalmente a errores humanos en el manejo de la sangre durante alguna etapa del proceso de obtención del componente sanguíneo transfundido. Se dice que esto puede ocurrir una vez por cada millón de unidades transfundidas ${ }^{14}$.

Existen múltiples factores que pueden afectar a una transfusión sanguínea y llevar a la ocurrencia de algún tipo de RAT. Ya se mencionó el caso de la contaminación bacteriana, pero otro factor es la transfusión masiva de hemocomponentes a un mismo paciente, que Maldonado-Rojas ${ }^{15}$ describe bastante bien.

Conocer el tipo de reacciones servirá como una herramienta para ayudar en su prevención. Es importante implementar un sistema de hemovigilancia para poder tener un mayor control en la seguridad y calidad en la atención al paciente.

\section{AGRADECIMIENTOS}

Al Servicio Transfusional del Hospital Regional de Alta Especialidad Ciudad Salud, por su colaboración.

\section{BIBLIOGRAFÍA}

1. Malagón MA, Berges GA, Bonifaz GR, Bravo LAG, Guerra MA, D'Artote GAL et al. Guía para el uso clínico de la sangre. Secretaría de Salud, Asociación Mexicana de Medicina Transfusional. México: AC Agrupación Mexicana para el Estudio de la Hematología; 2007.

2. Kumar P, Thapliyal R, Coshic P, Chatteriee K. Retrospective evaluation of adverse transfusion reactions following blood product transfusion from a tertiary care hospital : A preliminary step towards hemovigilance. Asian $J$ Transfus Sci. 2013;7(2):109-16.

3. Bolton MP, Cohen H. Serious Hazards of Transfusion (SHOT) haemovigilance and progress is improving transfusion safety. $\mathrm{Br} \mathrm{J}$ Haematol. 2013;163(3):303-14

4. Alter HJ, Klein HG. The hazards of blood transfusion in historical perspective. Blood. 2008;112(7):2617-26.

5. Kato H, Nakayama T, Uruma M, Okuyama Y, Handa M, Tomiyama Y, et al. A retrospective observational study to assess adverse transfusion reactions of patients with and without prior transfusion history. Vox Sang. 2015;108(3):243-50

6. Zimring JC, Spitalnik SL. Pathobiology of transfusion reactions. Annu Rev Pathol. 2015;10(1):83-110.

7. Linares RV, Díaz CS, Sánchez GS. Hemovigilancia: reacciones adversas a la transfusión en el Instituto Nacional de Cancerología. UNAM; 2014.

8. Gutiérrez CP, García GJ, Zarazúa TP, Rojo J. Reacciones transfusionales en el Hospital General de México. Revisión retrospectiva de cinco años. Rev Med Hosp Gen Mex. 2007;70(2):67-72.

9. Soporte de IBM. International Business Machines Corporation, EE.UU. [Internet]. Disponible en: http://www-01.ibm.com/support/docview.wss ?uid=swg24038592

10. Timler D, Klepaczka J, Kasielska TA, Bogusiak K. Analysis of complications after blood components' transfusions. Pol Przegl Chir. 2015;87(4):166-73.

11. Martínez MA, Rivero JA, Norma D, Fernández DN. Efectos adversos en la cadena transfusional en el Instituto de Hematología e Inmunología Revista Cubana de Hematol, Inmunol y Hemoter. 2015;31(3):288-300.

12. Cho J, Choi SJ, Kim S, Alghamdi E, Kim HO. Frequency and pattern of noninfectious adverse transfusion reactions at a tertiary care hospital in Korea. Ann Lab Med. 2016;36(1):36-41.

13. Philip J, Pawar A, Chatterjee T, Mallhi RS, Biswas AK, Dimri U. Non Infectious Complications Related to Blood Transfusion: An 11 year Retrospective Analysis in a Tertiary Care Hospital. Indian J Hematol Blood Transfus. 2016;32(3):292-8.

14. Rogers MA, Rohde JM, Blumberg N. Haemovigilance of reactions associated with red blood cell transfusion: comparison across 17 Countries. Vox Sang. 2016;110(3):266-77.

15. Maldonado Rojas M, Piña Farías L, Vásquez Rojas M, Toro Opazo C. Massive transfusion and complications associated. Rev Cubana Hematol Inmunol Hemoter. 2013;29(3):246-58. 